\title{
Serum N-3 Polyunsaturated Fatty Acid Levels Correlate With the Extent of Coronary Plaques and Calcifications in Patients With Acute Myocardial Infarction
}

\author{
Masayuki Ueeda, MD; Takenori Doumei, MD; Yoichi Takaya, MD; Ryoko Shinohata, MT*; \\ Yusuke Katayama, MD; Nobuhiko Ohnishi, MD; Atsushi Takaishi, MD; \\ Toru Miyoshi, MD**; Satoshi Hirohata, MD**; Shozo Kusachi, MD*
}

\begin{abstract}
Background The relationship between serum fatty acid levels and the extent of coronary plaques and calcification was examined in patients with acute myocardial infarction (AMI).

Methods and Results The serum levels of the n-3 polyunsaturated fatty acids (eicosapentaenoic acid (EPA) and docosahexaenoic acid (DHA)) and the n-6 polyunsaturated fatty acids (arachidonic acid (AA) and dihomogamma-linolenic acid (DGLA)) were determined using gas chromatography on admission of 95 consecutive patients with their first AMI and 17 controls. Using multidetector-row computed tomography, soft plaques and calcification lesions were scored according to the extent of coronary involvement. Serum logarithmic transformed $(\log )$ EPA and $\log$ DHA levels were inversely correlated with soft plaque scores $(r=-0.546, p<0.0001$ and $r=$ $-0.377, \mathrm{p}<0.0001$, respectively). Serum $\log \mathrm{AA}$ and $\log$ DGLA levels were not significantly correlated with soft plaque scores. Serum $\operatorname{logEPA}$ and $\log$ DHA levels were significantly, but weakly, correlated with calcification scores. Multivariate analysis with clinical characteristics and risk factors selected serum n-3 polyunsaturated fatty acid levels as independent factors associated with the extent of coronary soft plaques.

Conclusion The present study demonstrates a significant correlation between serum n-3 polyunsaturated fatty acid levels and the extent of coronary soft plaques and calcification in AMI patients. (Circ J 2008; 72: 1836-
\end{abstract} 1843)

Key Words: Atherosclerosis; Coronary artery disease; Fatty acids; Infarction; Risk factors

$\mathbf{P}$ rogression of coronary atherosclerosis and rupture of unstable plaque are key processes in acute myocardial infarction (AMI). The extent of coronary artery disease has been well documented as a major prognostic factor in patients with AMI. Prevention and evaluation of the progression of atherosclerotic plaque volume is thus essential to both reduce cardiac events and estimate prognosis after AMI.

Evidence that supplementation with n-3 polyunsaturated fatty acids prevents atherosclerosis-related cardiovascular risk has been accumulating.,2 More recently, a large randomized study demonstrated that eicosapentaenoic acid (EPA) is effective in preventing major coronary events in patients with hyperlipidemia? Dysfunction of the vascular endothelium, fatty streak formation and fibrous cap formation are processes in the formation of atherosclerotic lesions that are regulated by the action of vasoactive molecules,

(Received March 14, 2008; revised manuscript received June 9, 2008; accepted June 24, 2008; released online September 24, 2008)

Division of Cardiovascular Medicine, Mitoyo General Hospital, Kagawa, *Department of Medical Technology, Okayama University Graduate School of Health Sciences and **Department of Molecular Biology and Biochemistry, Okayama University Graduate School of Medicine, Dentistry and Pharmaceutical Sciences, Okayama, Japan Mailing address: Shozo Kusachi, MD, Department of Medical Technology, Okayama University Graduate School of Health Sciences, 2-5-1 Shikata-cho, Okayama 700-8558, Japan. E-mail: sh-ksc56@ pol.oninet.ne.jp

All rights are reserved to the Japanese Circulation Society. For permissions, please e-mail: cj@j-circ.or.jp growth factors, cytokines, and lipid metabolites. Several studies have demonstrated that supplementation with n-3 polyunsaturated fatty acids attenuates the exaggerated platelet aggregation induced by various stimuli 4,5 attenuates the action of growth factors and cytokines, ${ }^{5-7}$ reduces the serum triglyceride concentration, 8,9 and increases high-density lipoprotein levels?

There are several methods of evaluating coronary atherosclerotic lesions. Coronary angiography is widely used as the gold-standard to determine coronary stenosis, but it can only detect a luminal reduction in stenosis, collateral patterns and the presence of dense calcification of the coronary arteries, not plaque quantity. Coronary angioscopy and intravascular ultrasound (IVUS) can provide information about plaque characteristics to some extent ${ }^{10-14}$ but the observation areas are somewhat restricted and the methods are quite invasive. Multidetector spiral computed tomography (MDCT) with high slice numbers is now providing sufficient time and spatial resolution power to detect coronary atherosclerosis! ${ }^{15,16}$ Its reliability in the determination of coronary plaque morphology has been recently demonstrated by studies using IVUS and histopathologic studies ${ }^{17,18}$ The advantages of MDCT are that it is relatively noninvasive compared with intracoronary angioscopy and IVUS, and has sufficient stability for observation of the entire coronary tree. Indeed, at present, MDCT is thought to be 1 of the best methods of evaluating coronary plaque characteristics.

Based on these lines of evidence, we hypothesized that serum n-3 polyunsaturated fatty acid levels would correlate with the extent of coronary atherosclerotic lesions. The 
Table 1 Patient Characteristics and Laboratory Findings on Admission

\begin{tabular}{|c|c|c|c|}
\hline & AMI patients & Controls & $p$ value \\
\hline No. of patients & 95 & 17 & \\
\hline Age (years) & $68.3 \pm 10.9$ & $69.9 \pm 6.6$ & NS \\
\hline Gender $(M / F)$ & $71 / 24$ & $12 / 5$ & NS \\
\hline \multicolumn{4}{|l|}{ Culprit lesion } \\
\hline$L A D / L C X / R C A$ & $44 / 11 / 40$ & _- & \\
\hline Onset to admission time & $7.3 \pm 11.3$ & - & \\
\hline \multicolumn{4}{|c|}{ Extent of coronary artery disease } \\
\hline $1 / 2 / 3$ vessels & $35 / 37 / 23$ & _- & \\
\hline \multicolumn{4}{|l|}{ Enzymatic infarct size } \\
\hline Maximal CK $(I U / L)$ & $3,246 \pm 2,609$ & - & \\
\hline Maximal CKMB (IU/L) & $239 \pm 200$ & - & \\
\hline \multicolumn{4}{|l|}{ Coronary risk factor } \\
\hline Hypertension (+/-) & $50 / 31$ & $11 / 6$ & NS \\
\hline Hyperlipidemia (+/-) & $71 / 24$ & $10 / 7$ & $N S$ \\
\hline Diabetes mellitus (+/-) & $22 / 57$ & $4 / 13$ & NS \\
\hline Obesity (+/-) & $16 / 63$ & $9 / 7$ & NS \\
\hline Smoking (+/-) & $32 / 48$ & $8 / 9$ & $N S$ \\
\hline \multicolumn{4}{|c|}{ Laboratory findings on admission } \\
\hline$T C(\mathrm{mg} / \mathrm{dl})$ & $209 \pm 39$ & $191 \pm 29$ & 0.093 \\
\hline$T G(m g / d l)$ & $121 \pm 100$ & $121 \pm 41$ & $N S$ \\
\hline$H D L-C(m g / d l)$ & $48 \pm 14$ & $56 \pm 11$ & $<0.05$ \\
\hline$L D L-C(m g / d l)$ & $136 \pm 34$ & $111 \pm 22$ & $<0.005$ \\
\hline $\operatorname{HbA} I_{c}(\%)$ & $6.1 \pm 1.6$ & $5.9 \pm 1.9$ & $N S$ \\
\hline
\end{tabular}

$A M I$, acute myocardial infarction; LAD, left descending coronary artery; LCX, left circumflex coronary artery; RCA, right coronary artery; $C K$, creatine kinase; $C K M B$, creatine kinase and isoenzyme $M B$; $T C$, total cholesterol; $T G$, triglyceride; $H D L-C$, high-density lipoprotein-cholesterol; $L D L$-C, low-density lipoprotein-cholesterol; Hb, hemoglobin.

composition of serum fatty acid has been less studied in this context in AMI patients. Regarding the serum fatty acid component, but not the free fatty acid component, 1 study suggested a relationship between the fatty acid component and risk of coronary artery disease ${ }^{19}$ Although 1 report examined the association between the free fatty acid fraction and risk of myocardial infarction ${ }^{20}$ no reports have examined the correlation between serum fatty acid levels and the extent of coronary atherosclerotic lesions in AMI patients. Accordingly, using 16-slice MDCT, we examined the relationship between serum fatty acid component levels and the extent of coronary atherosclerotic lesions in patients with their first AMI.

\section{Methods}

\section{Patients}

A total of 95 consecutive patients with their first AMI who underwent successful reperfusion with percutaneous coronary intervention by means of coronary angioplasty with or without stenting and with subsequent adequate MDCT image collection were examined. Patients with cardiogenic shock and disturbed liver disorders were excluded. MDCT exclusion criteria were allergy to the contrast medium proven at the time of angiography, disturbed renal function and cardiac arrhythmia. Diagnosis of AMI was defined as the presence of ST-segment elevation $>0.1 \mathrm{mV}$ in more than 2 adjacent leads, associated with typical severe chest pain. Diagnosis was confirmed by the presence of a greater than 2-fold elevation in both serum creatine kinase and isoenzyme MB compared with the normal upper limit during the patient's clinical course. In addition to the 95 patients with AMI, 17 patients in whom MDCT showed no plaque and no calcification (soft plaque score 0 and calcification score 0 ; scoring system described later) served as controls. Controls were suspected to have coronary artery disease with an intact coronary artery determined by coro- nary angiography. In addition to these controls, we measured serum fatty acid levels in 30 age- and gender-matched people who underwent a medical check in the clinics. Further, we referred to serum levels of fatty acid component levels from a large population sample to confirm the validity of the control value. ${ }^{19}$ The clinical and angiographic characteristics of these patients are summarized in Table 1. The study complied with the rules of the Helsinki Declaration and informed consent was given by all patients and age- and gender-matched subjects before measurements of serum fatty acid levels. The study was approved by the institutional ethics committee for human research?

\section{Samples}

A blood sample was taken on admission. The time to blood sampling from last food ingestion was determined by interview and was $>3 \mathrm{~h}$ in all patients. Samples were separated by centrifugation at $2,500 \mathrm{~g}$ for $10 \mathrm{~min}$ and immediately stored at $-80^{\circ} \mathrm{C}$ until analysis.

\section{Serum Fatty Acids}

Fatty acid analysis has been described in detail elsewhere? 20,22 Lipids were extracted from serum by chloroform/ methanol extraction,23,24 with some modifications. Tricosanoic acid was used as an internal standard ${ }^{25}$ Methylation of fatty acids was carried out as described by Hoshi et al?6 Fatty acid composition in the methylated sample was analyzed by a gas chromatograph (HP5890A, Agilent Technologies Inc, Santa Clara, CA, USA) equipped with an auto-sampler (HP7673, Agilent Technologies Inc) and a data analyzer (C-R7A, Shimadzu Corporation, Kyoto, Japan). A capillary column (TC-WAX, GL Scinenses, Tokyo, Japan) was used. We determined the levels of the n-3 polyunsaturated fatty acids (EPA and docosahexaenoic acid (DHA)) and the n-6 polyunsaturated fatty acids (arachidonic acid (AA) and dihomo-gamma-linolenic acid (DGLA)). The extraction rate from samples with various quantities of added fatty 

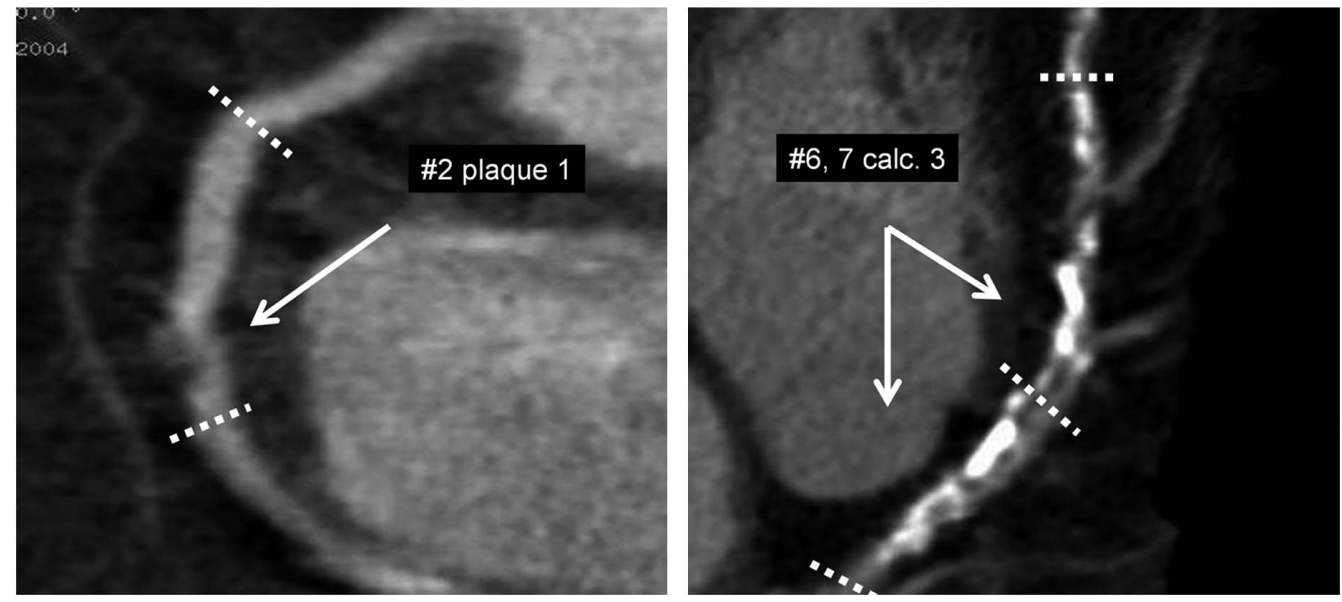

Fig 1. Representative multidetector spiral computed tomography coronary images. Soft plaque grading in segment 2 (grade 1: Left panel) and calcification grading in segments 6 and 7 (grade 3: Right panel) are shown.

acids was $91-100 \%$. The intra- and interassay coefficients of variation in the fatty acid measurements were 2.59 and $5.48 \%$, respectively. The ratio of $n-3$ polyunsaturated fatty acids to $n-6$ polyunsaturated fatty acids, in addition to the serum concentrations of each fatty acid component, was used in the statistical analysis to examine the relation between fatty acid and the extent of coronary atherosclerotic lesions.

\section{MDCT Scan Protocol}

MDCT was performed using a 16-detector slice computed tomograph (LightSpeed Ultra 16, GE Medical System, Boston, MA, USA) 5-7 days after admission, and the 16 slices were read out simultaneously in the cardiac mode. Contrast-enhanced scans of the coronary arteries were acquired using the following protocol: a total of $100 \mathrm{ml}$ of intravenous contrast agent $(300 \mathrm{mg} / \mathrm{ml}$ iodine $)$ was administered at $3 \mathrm{ml} / \mathrm{s}$ using $16 \times 0.75 \mathrm{~mm}$ collimation, a table feed with $3.8 \mathrm{~mm} /$ rotation, and a tube current of $650 \mathrm{~mA}$ at $120 \mathrm{kV}$. Computed tomography started at the aortic root cranial to the coronary ostia and stopped at the diaphragm caudal to all cardiac structures. ECG pulsing with a reduced tube current during systole was recorded during all scans to minimize radiation exposure.

\section{Image Reconstruction}

Axial images were reconstructed by the standard built-in ECG gated half or multisector scan reconstruction algorithm (temporal resolution 185 inches, slice thickness $1.0 \mathrm{~mm}$, increment $0.5 \mathrm{~mm}$, kernel B $30 \mathrm{f}$ ). Coronary images were reconstructed using volume-rendering and multiplanar reconstruction methods.

\section{MDCT Image Interpretation}

Evaluation of the MDCT images was performed by 2 cardiologists who were unaware of any clinical or angiographic data. Data were analyzed on an offline workstation for postprocessing (Advantage WS 4.1, GE Japan, Tokyo, Japan). The readers assessed 13 coronary segments from each patient according to a modified American Heart Association classification (right coronary artery $=$ segments $1-4$; left main $=$ segment 5 ; left anterior descending coronary artery = segments 6-10; left circumflex artery =segments 11-13). In accordance with the findings reported by Schroeder et al, ${ }^{18}$ coronary soft plaques were defined as lesions in which the density was attenuated from that of the contrastenhanced vessel lumen (density $<100$ Hounsfield U (HU)). A preliminary study was carried out to examine the agreement between the visual assessment of density attenuation and decrease in $\mathrm{HU}$ based on gradation gray scale analysis of the MDCT images. Calcified lesions were defined as lesions that showed apparently higher density (density $>130 \mathrm{HU}$ in native scans) than the contrast-enhanced vessel lumen. The extent of soft plaques was graded in each major coronary segment (segments 1,2,3,5, 6, 7, 11, 13 according to the American Heart Association classification) as follows: grade 0 , no soft plaque observed; 1 , soft plaque observed occupying $<1 / 3$ of the segment; 2 , soft plaque observed occupying between $1 / 3$ and $2 / 3$ of the segment; and 3 , diffusely observed soft plaque occupying the entire segment (Fig 1). Plaque score was determined as the sum of the grades obtained from each segment. Similarly, the calcification grade in each segment was determined as follows: 0, no calcification; 1 , calcification observed in $<1 / 3$ of the segment; 2 , calcification observed in $1 / 3$ to $2 / 3$ of the segment; and 3 , calcification observed diffusely throughout the entire segment. The calcification score was calculated as the sum of calcification grades for each segment. Inter- and intraobserver differences were checked for $10 \mathrm{MDCT}$ images.

\section{Statistical Analysis}

Kappa statistics were used to evaluate the reliability of intra- and interobserver agreement in the grading of coronary plaques and calcification. A chi-square fitness test clarified that serum fatty acid levels showed common logarithmic normal distribution and serum common logarithmic transformed (log) fatty acid values were thus used for statistical analysis. To elucidate the effects of the onset-sampling time on each fatty acid component level, simple and multiple correlation analyses was performed. In the multivariate analysis, stepwise linear regression analysis was performed using each serum fatty acid level as a dependent variable. Independent variables were onset-sampling time, age, gender, soft plaque score, calcification score and serum maximal creatinine kinase level. To determine the correlations of serum log fatty acid levels with coronary soft plaque scores and calcification scores, we used Spearman's rank correlation analysis. Pearson's correlation analysis was also used 
Table 2 Serum Fatty Acid Component Levels on Admission in AMI Patients and Controls, and Reported Averaged Levels in Population Samples

\begin{tabular}{|c|c|c|c|c|c|c|c|c|}
\hline \multirow{2}{*}{ Fatty acid } & \multirow{2}{*}{$\begin{array}{l}\text { AMI patients } \\
\quad(n=95)\end{array}$} & \multirow{2}{*}{$\begin{array}{l}\text { Controls } \\
(n=17)\end{array}$} & \multirow{2}{*}{$\begin{array}{l}p \text { value } \\
\text { (vs AMI) }\end{array}$} & \multirow{2}{*}{$\begin{array}{c}\text { Age- and } \\
\text { gender-matched } \\
\text { subjects } \\
(n=30)\end{array}$} & \multirow{2}{*}{$\begin{array}{l}p \text { value } \\
\text { (vs control) }\end{array}$} & \multicolumn{3}{|c|}{$\begin{array}{l}\text { Reported average levels } \\
\text { from population samples }{ }^{19}\end{array}$} \\
\hline & & & & & & $\begin{array}{l}\text { Commercial } \\
\text { district }\end{array}$ & $\begin{array}{c}\text { Farming } \\
\text { district }\end{array}$ & $\begin{array}{l}\text { Fishing } \\
\text { district }\end{array}$ \\
\hline $\log E P A$ & $1.782 \pm 0.219$ & $2.035 \pm 0.192$ & $<0.0001$ & $1.944 \pm 0.130$ & $N S$ & 1.952 & 1.922 & 1.986 \\
\hline $\log D H A$ & $2.151 \pm 0.129$ & $2.223 \pm 0.130$ & 0.0687 & $2.241 \pm 0.018$ & $N S$ & 2.274 & 2.243 & 2.243 \\
\hline $\log E P A+\log D H A$ & $3.933 \pm 0.318$ & $4.258 \pm 0.283$ & 0.0004 & & & & & \\
\hline $\log A A$ & $2.182 \pm 0.118$ & $2.184 \pm 0.086$ & NS & $2.234 \pm 0.090$ & $N S$ & 2.190 & 2.152 & 2.117 \\
\hline LogDGLA & $1.522 \pm 0.147$ & $1.466 \pm 0.159$ & $N S$ & $1.535 \pm 0.150$ & $N S$ & 1.543 & 1.565 & 1.490 \\
\hline $\log A A+\log D G L A$ & $3.704 \pm 0.232$ & $3.649 \pm 0.220$ & $N S$ & & & & & \\
\hline $\log A A / \log E P A$ & $1.241 \pm 0.152$ & $1.080 \pm 0.088$ & $<0.0001$ & & & & & \\
\hline $\log A A / \log D H A$ & $1.016 \pm 0.063$ & $0.984 \pm 0.035$ & 0.0266 & & & & & \\
\hline LogDGLA/LogEPA & $0.867 \pm 0.137$ & $0.726 \pm 0.102$ & $<0.0001$ & & & & & \\
\hline $\log D G L A / \log D H A$ & $0.709 \pm 0.066$ & $0.659 \pm 0.057$ & 0.0051 & & & & & \\
\hline$(\log A A+\log D G L A) / \log E P A$ & $2.108 \pm 0.278$ & $1.806 \pm 0.179$ & $<0.0001$ & & & & & \\
\hline$(\log A A+\log D G L A) / \log D H A$ & $1.725 \pm 0.111$ & $1.643 \pm 0.068$ & 0.0018 & & & & & \\
\hline $\log A A /(\log E P A+\log D H A)$ & $0.557 \pm 0.045$ & $0.514 \pm 0.023$ & 0.0001 & & & & & \\
\hline $\log G L A /(\log E P A+\log D H A)$ & $0.389 \pm 0.046$ & $0.345 \pm 0.037$ & 0.0003 & & & & & \\
\hline$(\log A A+\log D G L A) /(\log E P A+\log D H A)$ & $0.947 \pm 0.084$ & $0.859 \pm 0.052$ & $<0.0001$ & & & & & \\
\hline
\end{tabular}

EPA, eicosapentaenoic acid; DHA, docosahexaenoic acid; AA, arachidonic acid; DGLA, dihomo-gamma-linolenic acid. Other abbreviation see in Table 1.

to determine the relation of serum log fatty acid component levels to other coronary risk factors, especially serum cholesterol and triglycerides. Stepwise linear multiple regression analysis was also performed, using the plaque score as a dependent variable. The independent variables used were one of the fatty acid components' serum log levels (EPA, DHA, EPA+DHA or AA/EPA), age, gender, HR and risk factors for arteriosclerosis, including association with diabetes mellitus, hyperlipidemia, chronic renal disease (serum creatinine), or obesity. Hypertension and hyperlipidemia were diagnosed in accordance with guidelines? ${ }^{27,28}$ All values are expressed as mean $\pm \mathrm{SD}$, and $\mathrm{p}<0.05$ was considered significant.

\section{Results}

Reliability of Plaque Score Determination

The preliminary study to examine the correlation between the visual assessment of density attenuation and the decrease in MDCT density thresholds confirmed that visual assessment could detect a density attenuation by Kappa statistics $(\mathrm{p}<0.0001$, agreement rate $>97 \%)$. In 50 sample coronary lesions, Kappa statistics showed a high agreement rate $(>95 \%)$ in inter- and intra-observer measurements $(\mathrm{p}<0.001)$. Further, as for disagreement, only 1 grade difference occurred and this difference did not affect the results of the relationship between serum fatty acid components and plaque scores. Representative results for the MDCT coronary images in 2 cases are shown in Fig 1.

\section{Serum Fatty Acid Levels}

Regarding onset-blood sampling time, there were no significant correlations by simple correlation analyses between onset-admission (blood sampling time) and each serum fatty acid component level. Multivariate analyses did not reveal onset-blood sampling time as a factor that correlated with each serum fatty acid component level. The subsequent analyses were thus not affected by onset-blood sampling time. The serum log levels of fatty acid components examined on admission are listed in Table 2. The levels from 17 controls with MDCT soft plaque and calcification scores of 0 were in accordance with age- and gender-matched subjects $(67.0 \pm 3.4$ years; 20 men, 10 women) from those who
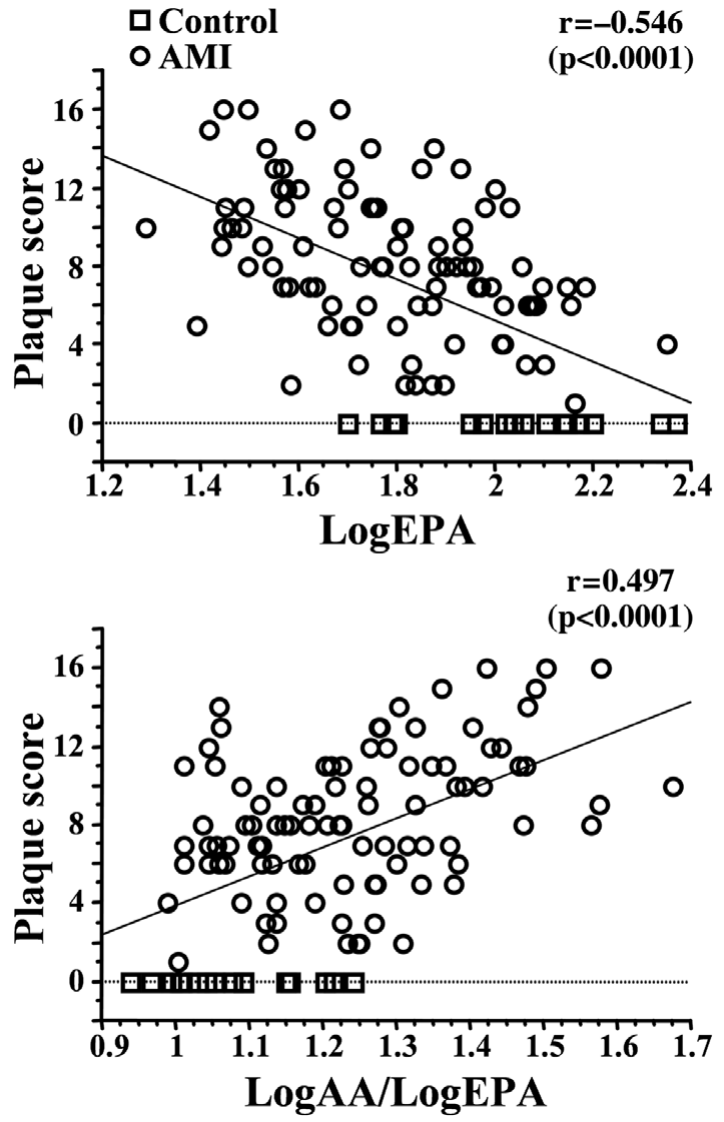

Fig 2. Correlations among serum logarithmic transformed (log) eicosapentaenoic acid (EPA) levels and the ratio of serum log levels of arachidonic acid (AA) to those of EPA $(\log \mathrm{AA} / \log \mathrm{EPA})$ with coronary plaque scores.

had a medical check-up in a clinic and with those obtained from large population samples in Kimihama, Japan. Significant differences in serum logEPA levels between AMI patients and controls were observed. LogDHA levels tended to show significant differences between AMI patients and controls. In contrast, there were no significant differences 
Table 3 Correlation of Serum Fatty Acid Component Levels With Plaque Scores

\begin{tabular}{|c|c|c|c|c|}
\hline \multirow{2}{*}{ Fatty acid } & \multicolumn{2}{|c|}{ Soft plaque score } & \multicolumn{2}{|c|}{ Calcification score } \\
\hline & $r$ & $p$ value & $r$ & $p$ value \\
\hline \multicolumn{5}{|l|}{$n 3$} \\
\hline $\log E P A$ & -0.546 & $<0.0001$ & -0.361 & 0.0001 \\
\hline $\log D H A$ & -0.377 & $<0.0001$ & -0.217 & 0.0221 \\
\hline $\log E P A+\log D H A$ & -0.536 & $<0.0001$ & -0.338 & 0.0004 \\
\hline \multicolumn{5}{|l|}{ n6 } \\
\hline $\log A A$ & -0.136 & $N S$ & -0.153 & $N S$ \\
\hline $\log D G L A$ & -0.009 & $N S$ & -0.027 & $N S$ \\
\hline $\log A A+\log D G L A$ & -0.064 & $N S$ & -0.083 & $N S$ \\
\hline \multicolumn{5}{|l|}{$n-6 / n-3$} \\
\hline $\log A A / \log E P A$ & 0.497 & $<0.0001$ & 0.321 & 0.0007 \\
\hline $\log A A / \log D H A$ & 0.256 & 0.007 & 0.070 & NS \\
\hline $\log D G L A / \log E P A$ & 0.406 & $<0.0001$ & 0.268 & 0.0048 \\
\hline $\log D G L A / \log D H A$ & 0.241 & 0.0112 & 0.122 & $N S$ \\
\hline$(\log A A+\log D G L A) / \log E P A$ & 0.473 & $<0.0001$ & 0.310 & 0.0011 \\
\hline$(\log A A+\log D G L A) / \log D H A$ & 0.271 & 0.0043 & 0.111 & $N S$ \\
\hline $\log A A /(\log E P A+\log D H A)$ & 0.443 & $<0.0001$ & 0.252 & 0.0078 \\
\hline $\log D G L A /(\log E P A+\log D H A)$ & 0.348 & 0.0002 & 0.214 & 0.0242 \\
\hline$(\log A A+\log D G L A) /(\log E P A+\log D H A)$ & 0.435 & $<0.0001$ & 0.266 & 0.0052 \\
\hline
\end{tabular}

Abbreviations see in Table 2.

Table 4 Stepwise Multiple Regression Analyses

\begin{tabular}{|c|c|c|c|c|c|c|}
\hline $\begin{array}{l}\text { Dependent } \\
\text { variables }\end{array}$ & $\begin{array}{l}\text { Selected } \\
\text { factors }\end{array}$ & $\begin{array}{l}\text { Regression } \\
\text { coefficient }\end{array}$ & $\begin{array}{l}\text { Standard error } \\
\text { of regression } \\
\text { coefficient }\end{array}$ & $\begin{array}{l}\text { Standardized } \\
\text { regression } \\
\text { coefficient }\end{array}$ & $\begin{array}{c}\text { Partial } \\
\text { correlation } \\
\text { coefficient }\end{array}$ & p value \\
\hline \multicolumn{7}{|c|}{ Analysis 1. Independent variables included serum EPA levels on admission as a fatty acid index with other clinical factors and serum lipids } \\
\hline \multirow[t]{5}{*}{ Plaque score } & $\log E P A$ & -10.4254 & 1.5533 & -0.5658 & -0.5886 & 0.00000 \\
\hline & Smoking & -2.8150 & 0.8030 & -0.3186 & -0.3554 & 0.00073 \\
\hline & $L D L-C$ & 0.0287 & 0.0109 & 0.2212 & 0.2748 & 0.01001 \\
\hline & Gender & 1.4377 & 0.9198 & 0.1416 & 0.1672 & 0.12175 \\
\hline & \multicolumn{6}{|c|}{ Multiple regression $r=0.637, p=0.00000$} \\
\hline \multicolumn{7}{|c|}{ Analysis 2. Independent variables included serum DHA levels on admission as a fatty acid index with other clinical factors and serum lipids } \\
\hline \multirow[t]{4}{*}{ Plaque score } & $\log D H A$ & -12.0784 & 3.3713 & -0.3484 & -0.3604 & 0.00056 \\
\hline & Smoking & -2.1217 & 0.8545 & -0.2401 & -0.2586 & 0.01498 \\
\hline & $L D L-C$ & 0.0272 & 0.0126 & 0.2102 & 0.2273 & 0.03317 \\
\hline & \multicolumn{6}{|c|}{ Multiple regression $r=0.446, p=0.00025$} \\
\hline \multicolumn{7}{|c|}{ Analysis 3. Independent variables included serum EPA + DHA levels on admission as a fatty acid index with other clinical factors and serum lipids } \\
\hline \multirow[t]{5}{*}{ Plaque score } & $\log E P A+\log D H A$ & -7.1943 & 1.1404 & -0.5442 & -0.5647 & 0.00000 \\
\hline & Smoking & -2.8222 & 0.8201 & -0.3194 & -0.3497 & 0.00090 \\
\hline & $L D L-C$ & 0.0296 & 0.0111 & 0.2285 & 0.2774 & 0.00929 \\
\hline & Gender & 1.4998 & 0.9396 & 0.1477 & 0.1706 & 0.11414 \\
\hline & \multicolumn{6}{|c|}{ Multiple regression $r=0.617, p=0.00000$} \\
\hline \multicolumn{7}{|c|}{ Analysis 4. Independent variables included serum AA/EPA levels on admission as a fatty acid index with other clinical factors and serum lipids } \\
\hline \multirow[t]{5}{*}{ Plaque score } & $\log A A / \log E P A$ & 13.7672 & 2.4120 & 0.5040 & 0.5264 & 0.00000 \\
\hline & Smoking & -2.6167 & 0.8425 & -0.2962 & -0.3192 & 0.00258 \\
\hline & $L D L-C$ & 0.0189 & 0.0114 & 0.1458 & 0.1766 & 0.10173 \\
\hline & Gender & 1.4055 & 0.9673 & 0.1384 & 0.1557 & 0.14992 \\
\hline & \multicolumn{6}{|c|}{ Multiple regression $r=0.585, p=0.00000$} \\
\hline
\end{tabular}

Abbreviations see in Tables 1,2.

in the serum log levels of n-6 polyunsaturated fatty acids, AA and DGLA, between AMI patients and controls. Ratios of serum log levels of n-6 polyunsaturated fatty acids to those of n-3 polyunsaturated fatty acids were significantly higher in AMI patients than controls.

\section{Correlation of Fatty Acid Component Levels With}

Plaque and Calcification Scores

Serum $\log$ EPA and $\log$ DHA levels were inversely correlated with soft plaque scores (Fig 2). The correlations between the serum log levels of n-3 and n-6 polyunsaturated fatty acid components and plaque scores, analyzed by Spearman's rank correlation analysis, are summarized in
Table 3. There were no significant positive correlations between the serum log levels of n- 6 polyunsaturated fatty acid components and soft plaque scores. Because regression coefficients in the relationship between serum $\log \mathrm{AA}$ or logDGLA level and plaque score were negative, correlations of the ratios of serum log levels of $n-6$ to those of $n-3$ polyunsaturated fatty acids with soft plaque scores were not higher than those between the serum log levels of n-3 polyunsaturated fatty acids and soft plaque scores. The results of stepwise multiple linear regression analysis are shown in Table 4. Serum $\log$ EPA, $\log$ DHA and $\operatorname{logEPA}+$ $\log$ DHA levels were independent factors significantly associated with an increase in plaque scores. 
Table 5 Correlation of Polyunsaturated Fatty Acids With Serum Lipids, UA and Plasma HbA1c Levels

\begin{tabular}{|c|c|c|c|c|c|c|c|c|c|c|c|c|}
\hline & \multicolumn{2}{|c|}{$T C$} & \multicolumn{2}{|c|}{$T G$} & \multicolumn{2}{|c|}{$H D L-C$} & \multicolumn{2}{|c|}{$L D L-C$} & \multicolumn{2}{|c|}{$U A$} & \multicolumn{2}{|c|}{$H b A l c$} \\
\hline & $r$ & $p$ value & $r$ & $p$ value & $r$ & $p$ value & $r$ & $p$ value & $r$ & $p$ value & $r$ & $p$ value \\
\hline \multicolumn{13}{|l|}{$n 3$} \\
\hline LogEPA & 0.286 & 0.0026 & 0.035 & $N S$ & 0.384 & $<0.0001$ & 0.145 & $N S$ & 0.086 & $N S$ & 0.117 & $N S$ \\
\hline $\log D H A$ & 0.467 & $<0.0001$ & 0.377 & $<0.0001$ & 0.258 & 0.0065 & 0.241 & 0.0116 & 0.157 & $N S$ & -0.007 & $N S$ \\
\hline $\log E P A+\log D H A$ & 0.377 & $<0.0001$ & 0.174 & 0.0674 & 0.358 & 0.0002 & 0.186 & 0.0516 & 0.120 & $N S$ & 0.094 & $N S$ \\
\hline \multicolumn{13}{|l|}{ n6 } \\
\hline $\log A A$ & 0.463 & $<0.0001$ & 0.216 & 0.0230 & 0.266 & 0.0051 & 0.308 & 0.0013 & -0.035 & NS & -0.070 & $N S$ \\
\hline $\log D G L A$ & 0.555 & $<0.0001$ & 0.498 & $<0.0001$ & 0.056 & $N S$ & 0.394 & $<0.0001$ & 0.076 & $N S$ & 0.110 & $N S$ \\
\hline $\log A A+\log D G L A$ & 0.580 & $<0.0001$ & 0.432 & $<0.0001$ & 0.146 & NS & 0.405 & $<0.0001$ & 0.027 & $N S$ & 0.042 & $N S$ \\
\hline
\end{tabular}

UA, uric acid. Other abbreviations see in Tables 1,2.

Table 6 Correlation of Serum Lipids, UA Levels and Plasma HbA1c Levels With Plaque and Calcification Score

\begin{tabular}{|c|c|c|c|c|c|c|c|c|c|c|c|c|}
\hline & \multicolumn{2}{|c|}{$T C$} & \multicolumn{2}{|c|}{$T G$} & \multicolumn{2}{|c|}{$H D L-C$} & \multicolumn{2}{|c|}{$L D L-C$} & \multicolumn{2}{|c|}{$U A$} & \multicolumn{2}{|c|}{$H b A l c$} \\
\hline & $r$ & $p$ value & $r$ & $p$ value & $r$ & $p$ value & $r$ & p value & $r$ & $p$ value & $r$ & $p$ value \\
\hline Plaque & -0.058 & $N S$ & -0.113 & $N S$ & -0.285 & 0.003 & 0.075 & $N S$ & 0.065 & $N S$ & -0.047 & $N S$ \\
\hline Calcification & -0.112 & $N S$ & -0.033 & $N S$ & -0.338 & 0.000 & -0.027 & $N S$ & 0.148 & $N S$ & -0.036 & $N S$ \\
\hline
\end{tabular}

Abbreviations see in Tables 1,5.

Serum $\log$ EPA, $\log$ DHA and sum of $\log$ EPA and $\log$ DHA levels were significantly correlated with coronary calcification scores (Table 3). Serum $\log$ AA, $\log$ DGLA and the sum of the $\log \mathrm{AA}$ and $\log$ DGLA levels were not significantly correlated with calcification scores. Some of the ratios of the serum log levels of $n-6$ to those of $n-3$ polyunsaturated fatty acids correlated with calcification scores, but the correlations were not higher than those in the relationship between $\mathrm{n}-3$ polyunsaturated fatty acids serum $\log$ levels and calcification scores. Multivariate analysis selected serum logEPA level as independently correlated with calcification score (partial correlation coefficient, $-0.262, \mathrm{p}=0.013$ ).

\section{Relationship of Serum Fatty Acid Components to Serum Lipid, Uric Acid and Hemoglobin Alc Levels}

Serum $\log$ EPA, $\log$ DHA, $\log$ AA and $\log$ DGLA levels were all significantly correlated with serum total cholesterol with weak to moderate correlation coefficients (Table 5). Serum $\log$ AA and $\log$ DGLA were significantly correlated with low-density lipoprotein-cholesterol (LDL-C). In contrast, serum $\operatorname{logEPA}$ and $\log \mathrm{DHA}$ levels were not, or very weakly, correlated with LDL-C. Serum log levels of n-3 polyunsaturated fatty acids were significantly correlated with high-density lipoprotein-cholesterol (HDL-C). Serum $\log$ levels of $n-6$ polyunsaturated fatty acids were not, or weakly, correlated with HDL-C compared to those of n-3 polyunsaturated fatty acids.

Correlation of Serum Lipid, Uric Acid and Hemoglobin Alc Levels With Plaque and Calcification Scores

Among the serum lipids, HDL-C levels were negatively correlated with coronary soft plaque and calcification scores (Table 6). Significant correlations between other serum biomarkers and coronary soft plaque or calcification scores were not obtained.

\section{Discussion}

The present study has demonstrated semi-quantitatively that in patients with their first AMI, the log serum n-3 polyunsaturated fatty acids levels are negatively correlated with the extent of coronary soft plaques and calcifications.

We assessed the extent of coronary atherosclerosis by MDCT. A previous study ${ }^{18}$ demonstrated a significant relationship between MDCT density thresholds and histopathologic plaque findings in postmortem-scanned hearts. That study used a modified Stary 8-stage system to classify coronary plaques into 3 groups according to their histopathologic characteristics: lipid-rich, intermediate and calcified 29 There were overlapping values for plaques density (HU) between lipid-rich and intermediate plaques, so differentiation between these 2 types of plaque was difficult. Therefore, we analyzed lipid-rich and intermediate plaques together as soft plaques. Conversely, detection of calcification was adequate by visual assessment because the increase in density was marked. We evaluated the degree of plaque based on the involvement of the coronary arteries, rather than the plaque density. The intra- and interobserver differences were acceptably small. Although the number of controls was relatively small, because MDCT cannot be performed in healthy subjects, the fatty acid levels from 17 controls with both soft plaque and calcification scores of 0 were in good agreement with those from the subjects in the clinics and with those obtained from large population samples. ${ }^{19}$ The controls were thus judged appropriate. These considerations indicate that our evaluation of the extent of coronary plaques and calcification was reasonable, and that the results of soft plaque scores and calcification scores could be analyzed with reference to serum fatty acid levels.

The methods used to measure serum fatty acids are well established $20,22,23,26$ Moreover, blood samples were not collected soon after eating. Conflicting results regarding the time course of free fatty acid levels after AMI have been reported. Several previous reports found an increase in total plasma free fatty acid levels ${ }^{30-32}$ while a relatively recent study did not find any significant increase? ? $^{2,33}$ Regarding fatty acids in serum or plasma, there have been no studies that examined time-dependent changes in total serum fatty acids. The percentage of free fatty acids in serum fatty acid is very low. Total serum fatty acid is thus thought not to be largely changed and the fraction of each fatty acid compo- 
nent to the total fatty acid is unlikely to be changed considerably after AMI. In fact, no correlation was observed by simple and multivariate correlation analyses between onsetadmission (blood sampling time) and each fatty acid component in the present study. Actual values of each serum fatty acid component were thus not largely different from the percentage of each fatty acid component to total fatty acid. Further discussion of the results can thus be made.

We measured the serum levels of the n-3 polyunsaturated fatty acids EPA and DHA, and the n- 6 polyunsaturated fatty acids DGLA and AA on admission in patients with AMI. To date, only one other study ${ }^{20}$ has examined the free fatty acid components in patients with AMI, demonstrating that the relative levels of DHA and EPA in the serum FFA fraction were significantly lower in AMI patients than in controls. Although our study examined serum total levels of each fatty acid component, but did not measure it in a free fatty acid fraction, the present results are not consistent with other studies regarding n-3 polyunsaturated fatty acids. Conversely, an increase in the relative levels of oleic and linoleic acid in the serum free fatty acid fraction of AMI patients was also observed in an earlier study. Because we measured DGLA and AA, not oleic and linoleic acid, and the earlier study obtained percentage values for both acids in the total free fatty acid, a precise comparison of the results regarding n- 6 polyunsaturated fatty acids cannot be made.

The present results demonstrate that serum n-3 polyunsaturated fatty acid levels are significantly correlated with the extent of coronary plaques and calcifications in AMI patients. There have been no previous studies reporting a quantitative relationship between serum fatty acid components and the extent of coronary plaques in AMI patients, so these results cannot be compared directly with previous reports. There have been several basic studies that examined the effects of n-3 fatty acids on processes involved in atherosclerosis. The reported effects are attenuation of platelet aggregation caused by various stimuli, decreases in triacylglycerol and cholesterol, and an increase in HDL-C? More recently, reductions in interleukin $1 \beta$ and tumor necrosis factor concentrations because of $n-3$ fatty acids have also been observed ex vivo, 5 ,6 Dietary $n-3$ fatty acids have also been shown to downregulate the expression of both PDGF-A and PDGF-B genes in the quiescent mononuclear cells of volunteers? indicating that a deliberate change in the diet can change human gene expression. N-3 fatty acids have been also reported to reduce blood pressure in patients with hypertension ${ }^{34-36}$ Several studies have indicated that low serum n-3 fatty acids are associated with risk of coronary artery disease ${ }^{37-39}$ On the other hand, Harris et al evaluated the relationship between fatty acid levels and coronary artery disease using a large amount of data from a case-controlled and prospective cohort study and revealed that omega-3 fatty acid levels correlated with coronary artery disease, indicating that the levels may have both prognostic and diagnostic utility in coronary artery disease risk. 40 The present findings of a significant correlation between the serum fatty acid profile and the extent of coronary plaques are completely in agreement with those basic and population-based studies. The present results showing a significant correlation between the serum n-3 polyunsaturated fatty acid levels and the extent of coronary plaques and calcifications imply that supplementation of $n-3$ polyunsaturated fatty acid may have favorable effects on the progression of coronary atherosclerosis? In fact, long-term use of highly purified EPA $(1,800 \mathrm{mg} /$ day) reduced cardiac events and consequently, n-3 polyunsaturated fatty acid is indicated for prevention of coronary artery disease.

No significant positive correlation between n-6 polyunsaturated fatty acid (AA and DGLA) levels and coronary plaque scores was obtained, so the n- $6 / n-3$ fatty acid ratio did not show a superior correlation in terms of the extent of coronary plaques. Although the ratio of omega- 6 fatty acids to omega- 3 fatty acids has been proposed in several reports as a biomarker of risk for coronary artery disease, analysis of a large data set from multiple studies concluded that neither the n- 6 fatty acid level nor the ratio of n-6/n-3 fatty acids is a useful biomarker for the risk of coronary artery disease. 40 The present results are in complete agreement with the results of previous large-scale data analysis.

Among the well-known risk factors, only serum HDL-C levels correlated weakly with the extent of coronary plaques and calcification scores in the present study. The reason for the insignificant correlation of other well-known risk factors, such as LDL-C, with coronary plaque scores and calcification was obscure and may be related to an insufficient number of patients. Our results suggest that serum fatty acid component levels may be a more useful risk factor for coronary atherosclerosis than other well-known risk factors. Basic studies have indicated an interaction between cholesterol and fatty acid and, in fact, the present study showed a significant, but weak, correlation between some well-known risk factors and some serum fatty acid components? The previous reported evidence, together with the present findings, indicates that serum fatty acid components reflect the risk for coronary artery disease; however, superiority between these 2 factors remains obscure because of the lack of concrete data.

Although we found significant correlations between the serum levels of n-3 polyunsaturated fatty acid and the soft plaque score, they were somewhat low. Coronary atherosclerotic processes comprise multiple steps with the involvement of multiple factors. The correlation of each factor with coronary atherosclerosis is not likely to be large and this is why the correlations between n-3 polyunsaturated fatty acid levels and coronary soft plaque scores were not high.

\section{Study Limitations}

First, it was a cross-sectional study and the relationship between serum fatty acid components and outcomes could not be analyzed. Second, patients with angina pectoris were not studied, but the present results warrant study of the relationship between the serum fatty acid profile and patients with angina pectoris.

\section{Conclusion}

The present study has demonstrated for the first time a significant correlation between the serum fatty acid profile and the extent of coronary plaques.

\section{Note}

There is neither a conflict of interest nor financial support in connection with the present study. The authors did not receive any of gifts, technical help, or other assistance.

\section{References}

1. von Schacky C. n-3 fatty acids and the prevention of coronary athero- 
sclerosis. Am J Clin Nutr 2000; 71: 224S-227S.

2. Bhatnagar D, Durrington PN. Omega-3 fatty acids: Their role in the prevention and treatment of atherosclerosis related risk factors and complications. Int J Clin Pract 2003; 57: 305-314.

3. Yokoyama M, Origasa H, Matsuzaki M, Matsuzawa Y, Saito Y, Ishikawa $\mathrm{Y}$, et al. Effects of eicosapentaenoic acid on major coronary events in hypercholesterolaemic patients (JELIS): A randomised open-label, blinded endpoint analysis. Lancet 2007; 369: 1090 1098 .

4. von Schacky C. Prophylaxis of atherosclerosis with marine omega-3 fatty acids: A comprehensive strategy. Ann Intern Med 1987; 107: $890-899$.

5. Endres S, von Schacky C. n-3 polyunsaturated fatty acids and human cytokine synthesis. Curr Opin Lipidol 1996; 7: 48-52.

6. Endres S, Ghorbani R, Kelley VE, Georgilis K, Lonnemann G, van der Meer JW, et al. The effect of dietary supplementation with n-3 polyunsaturated fatty acids on the synthesis of interleukin-1 and tumor necrosis factor by mononuclear cells. N Engl J Med 1989; 320: $265-271$.

7. Ross R. The pathogenesis of atherosclerosis: A perspective for the 1990s. Nature 1993; 362: 801-809.

8. Harris WS. Fish oils and plasma lipid and lipoprotein metabolism in humans: A critical review. J Lipid Res 1989; 30: 785-807.

9. Moreno JJ, Mitjavila MT. The degree of unsaturation of dietary fatty acids and the development of atherosclerosis (Review). J Nutr Biochem 2003; 14: 182-195.

10. Asakura M, Ueda Y, Yamaguchi O, Adachi T, Hirayama A, Hori M, et al. Extensive development of vulnerable plaques as a pan-coronary process in patients with myocardial infarction: An angioscopic study. J Am Coll Cardiol 2001; 37: 1284-1288.

11. Thieme T, Wernecke KD, Meyer R, Brandenstein E, Habedank D, Hinz A, et al. Angioscopic evaluation of atherosclerotic plaques: Validation by histomorphologic analysis and association with stable and unstable coronary syndromes. J Am Coll Cardiol 1996; 28: 1-6.

12. Hodgson JM, Reddy KG, Suneja R, Nair RN, Lesnefsky EJ, Sheehan HM. Intracoronary ultrasound imaging: Correlation of plaque morphology with angiography, clinical syndrome and procedural results in patients undergoing coronary angioplasty. $J$ Am Coll Cardiol 1993; 21: 35-44.

13. Kato M, Dote K, Sasaki S, Ueda K, Matsuda O, Nakano Y, et al Coronary plaque vulnerability in metabolic syndrome: Assessment of carotid artery morphology in acute coronary syndrome. Circ J 2007; 71: $1229-1233$.

14. Okura H, Taguchi H, Kubo T, Toda I, Yoshida K, Yoshiyama M, et al. Atherosclerotic plaque with ultrasonic attenuation affects coronary reflow and infarct size in patients with acute coronary syndrome: An intravascular ultrasound study. Circ J 2007; 71: 648-653.

15. Kuettner A, Beck T, Drosch T, Kettering K, Heuschmid M, Burgstahler $\mathrm{C}$, et al. Image quality and diagnostic accuracy of noninvasive coronary imaging with 16 detector slice spiral computed tomography with $188 \mathrm{~ms}$ temporal resolution. Heart 2005; 91: 938 941.

16. Hara T, Yamada S, Hayashi T, Ikeda Y, Yamashiro K, Mizutani K, et al. Accuracy of nonstenotic coronary atherosclerosis assessment by multi-detector computed tomography. Circ J 2007; 71: 911-914.

17. Komatsu S, Hirayama A, Omori Y, Ueda Y, Mizote I, Fujisawa Y, et al. Detection of coronary plaque by computed tomography with a novel plaque analysis system, 'Plaque Map', and comparison with intravascular ultrasound and angioscopy. Circ J 2005; 69: 72-77.

18. Schroeder S, Kuettner A, Leitritz M, Janzen J, Kopp AF, Herdeg C, et al. Reliability of differentiating human coronary plaque morphology using contrast-enhanced multislice spiral computed tomography: A comparison with histology. J Comput Assist Tomogr 2004; 28: 449_ 454.

19. Nakamura T, Azuma A, Kuribayashi T, Sugihara H, Okuda S, Nakagawa M. Serum fatty acid levels, dietary style and coronary heart disease in three neighbouring areas in Japan: The Kumihama study. Br J Nutr 2003; 89: 267-272.
20. Yli-Jama P, Meyer HE, Ringstad J, Pedersen JI. Serum free fatty acid pattern and risk of myocardial infarction: A case-control study. J Intern Med 2002; 251: 19-28.

21. World Medical Association Declaration of Helsinki. Recommendations guiding physicians in biomedical research involving human subjects. Cardiovasc Res 1997; 35: 2-3.

22. Ozawa T, Takayanagi K, Fujita T, Hirai A, Hamazaki T, Terano T, et al. Determination of long chain fatty acids in human total plasma lipids using gas chromatography. Bunseki Kagaku 1981; 31: 87-91 (in Japanese with English abstract).

23. Kaluzny MA, Duncan LA, Merritt MV, Epps DE. Rapid separation of lipid classes in high yield and purity using bonded phase columns. J Lipid Res 1985; 26: 135-140.

24. Folsh J, Lees M, Sloane M, Stanley G. A simple method for the isolation and purification of total lipids from animal tisues. J Biol Chem 1957; 226: 497-509.

25. Shantha NC, Ackman RG. Nervonic acid versus tricosanoic acid as internal standards in quantitative gas chromatographic analyses of fish oil longer-chain n-3 polyunsaturated fatty acid methyl esters. J Chromatogr 1990; 533: 1-10.

26. Hoshi M, Williams M, Kishimoto Y. Esterification of fatty acids at room temperature by chloroform-methanolic $\mathrm{HCl}$-cupric acetate. J Lipid Res 1973; 14: 599-601.

27. The sixth report of the Joint National Committee on prevention, detection, evaluation, and treatment of high blood pressure. Arch Intern Med 1997; 157: 2413-2446.

28. Hata Y, Mabuchi H, Saito Y, Itakura H, Egusa G, Ito H, et al. Report of the Japan Atherosclerosis Society (JAS) Guideline for Diagnosis and Treatment of Hyperlipidemia in Japanese adults. $J$ Atheroscler Thromb 2002; 9: 1-27.

29. Stary HC. Natural history and histological classification of atherosclerotic lesions: An update. Arterioscler Thromb Vasc Biol 2000; 20: $1177-1178$

30. Crofts JW, Ogburn PL Jr, Johnson SB, Holman RT. Polyunsaturated fatty acids of serum lipids in myocardial infarction. Lipids 1988; 23: $539-545$.

31. Gupta DK, Jewitt DE, Young R, Hartog M, Opie LH. Increased plasma-free-fatty-acid concentrations and their significance in patients with acute myocardial infarction. Lancet 1969; 2: 1209-1213.

32. Flink EB, Brick JE, Shane SR. Alterations of long-chain free fatty acid and magnesium concentrations in acute myocardial infarction. Arch Intern Med 1981; 141: 441-443.

33. Hagenfeldt L, Wester PO. Plasma levels of individual free fatty acids in patients with acute myocardial infarction. Acta Med Scand 1973; 194: $357-362$

34. Knapp HR, FitzGerald GA. The antihypertensive effects of fish oil: A controlled study of polyunsaturated fatty acid supplements in essential hypertension. N Engl J Med 1989; 320: 1037-1043.

35. Bonaa KH, Bjerve KS, Straume B, Gram IT, Thelle D. Effect of eicosapentaenoic and docosahexaenoic acids on blood pressure in hypertension: A population-based intervention trial from the Tromso study. N Engl J Med 1990; 322: 795-801.

36. Toft I, Bonaa KH, Ingebretsen OC, Nordoy A, Jenssen T. Effects of n-3 polyunsaturated fatty acids on glucose homeostasis and blood pressure in essential hypertension: A randomized, controlled trial. Ann Intern Med 1995; 123: $911-918$.

37. Riemersma RA, Wood DA, Butler S, Elton RA, Oliver M, Salo M, et al. Linoleic acid content in adipose tissue and coronary heart disease. BMJ (Clin Res Ed) 1986; 292: 1423-1427.

38. Oliver MF, Riemersma RA, Thomson M, Fulton M, Abraham RA, Wood DA. Linoleic acid and coronary heart disease. Br J Hosp Med 1989; 42: 298, 301-292.

39. Kondo T, Ogawa K, Satake T, Kitazawa M, Taki K, Sugiyama S, et al. Plasma-free eicosapentaenoic acid/arachidonic acid ratio: A possible new coronary risk factor. Clin Cardiol 1986; 9: 413-416.

40. Harris WS, Assaad B, Poston WC. Tissue omega-6/omega-3 fatty acid ratio and risk for coronary artery disease. Am J Cardiol 2006; 98: $19 \mathrm{i}-26 \mathrm{i}$ 